\title{
GRAVIDEZ NA ADOLESCÊNCIA: ATUAÇĀO DA ENFERMEIRA
}

\author{
Anna Maria Hecker Luz * \\ Sandra Maria de Abreu Mendes ** \\ Sonia Maria Motink Agostini *
}

$\operatorname{ReBEn} / 01$

MENDES, S.M.A. e Colaboradoras - Gravidez na Adolescência: Atuação da Enfermeira. Rev. Bras. Enf.: RS. 36: 3-12, 1983.

\begin{abstract}
RESUMO
O presente trabalho foi efetuado considerando nossa preocupação relativa à problemática mundial em torno da adolescente, principalmente no que se refere ao aumento da incidência de gravidez nessa faixa etária em outros países, como também a ausência de serviços específicos de atendimento a esta clientela em Porto Alegre. Para tanto, foi realizado um estudo retrospectivo de 1.597 prontuários de parturientes atendidas em um Hospital-Escola da cidade, onde ficou evidenciado o percentual de $12,39 \%$ de parturientes adolescentes. Tentamos caracterizar, através deste trabalho, a problemática das adolescentes grávidas relacionada ao: estado civil, ocupação, época da menarca, in ício das relações sexuais, gestações e paridade, abortamento, métodos anticoncepcionais, freqüência pré-natal e a atuação da enfermeira no atendimento da parturiente adolescente.
\end{abstract}

\section{I - INTRODUÇĀO} adolescência.

$\mathrm{Na}$ literatura mundial, cada vez mais encontramos trabalhos relacionados à gravidez na

Em nossa atividade profissional verificamos que não só a literatura indicava, mas também nos parecia que a incidência de adolescentes grávidas aumentava em nosso meio.

A adolescência é um processo psicológico social e de amadurecimento iniciado na puberdade. Durante esta fase, a jovem adolescente torna-se consciente das mudanças em seu corpo, sofre emoções que vão do orgulho à vergonha e ansiedade, e, frente à reação dos outros à s suas mudanças, começa a formular nova identidade própria: gravidez neste período pode acrescentar pesada carga emocional, física e social.

Surpreendeu-nos a inexistência de serviços específicos voltados para o atendimento da jovem justamente no momento em que ela apresenta tantas mudanças em seu desenvolvimento.

* Professora Assistente do Departamento de Enfermagem Materno-Infantil da Escola de Enfermagem da Universidade Federal do Rio Grande do Sul.

* *Professora Adjunto do Departamento de Enfermagem Materno-Infantil da Escola de Enfermagem da Universidade Federal do Rio Grande do Sul.

Assessora do Serviço de Enfermagem Materno Infantil do Hospital de Clínicas de Porto Alegre. 
MENDES, S.M.A. e Colaboradoras - Gravidez na Adolescência: Atuação da Enfermeira. Rev. Bras Enf.: RS. 36: 3-12, 1983.

A finalidade deste trabalho é dar início a uma série de estudos visando uma caracterização mais precisa desta problemática entre nós. Para tanto, procuramos verificar através desta pesquisa:

- a incidência de adolescentes grávidas em nosso meio;

- as características da população em estudo, tais como estado civil, ocupação, época da menarca, início das relações sexuais, gestações, paridade, abortamento, anticoncepção e freqüência pré-natal;

- a atuação da enfermeira, em termos assistenciais, junto à parturiente adolescente.

\section{II - REVISĀO DA LITERATURA}

Existem hoje numerosos trabalhos mostrando a importância da atenção que deve ser dada à adolescente grávida e a todas as implicações da gravidez nos jovens. Citaremos alguns dos que mais se situem na problemática que o presente estudo busca abordar.

A combinação da maturidade sexual precoce e do casamento tardio, e as maiores oportunidades de contatos sexuais proporcionados pelo estilo de vida moderno, fazem com que a gravidez e a maternidade, no mundo inteiro, ocorram em grupos etários mais jovens do que no passado ${ }^{13}$.

Segundo HUFFMAN e colaboradores ${ }^{9}$, a tendência de que a maturação ocorra em idade antecipada se tem feito sentir nos últimos 75 a 100 anos.

Para BABIKIAN 2 , a maternidade da adolescente apresenta problemas, tais como: a predisposição da jovem a complicações obstétricas, tensões fisiológicas e emocionais provenientes da gravidez, ao mesmo tempo que tem que suportar as perturbações criadas no ambiente familiar.

KLAUS1 1 descobriu que, enquanto muitas adolescentes haviam, a princípio, encarado a gravidez como um meio de obter atenção e se fazerem sentir "crescidas" e importantes ao final sentem-se sós, desamparadas, amedrontadas e aborrecidas.

CURTIS6, observando mães adolescentes, concluiu: que são meninas solitárias, amedrontadas, apanhadas em ciclo de desânimo e privação, que, sem alguma intervenção, pode se perpetuar. Diz ainda que são garotas que não desenvolveram confian ’a e muitas vezes usam mecanismos de defosa, tais como hostilidade e desafio à autoridade. Muitas vezes, o medo de perder o namorado foi o motivo para o início das relações sexuais, e a maioria delas fantasiava que isso seria o primeiro passo para um futuro brilhante.

PIERRE15, em estudos realizados, verificou que socialmente a adolescente grávida gira em um ciclo de baixa realização educacional, nível de emprego inferior e subseqüente renda mais baixa. Para aquelas que casam, os casamentos são freqüentemente instáveis. De cinco noivas adolescentes grávidas, três divorciam-se dentro de seis anos. Estas jovens adolescentes subsequientemente têm filhos mais rapidamente e em maior número do que as mulheres que têm filhos com mais idade. Esta rápida sucessão de gravidezes exarceba o problema.

POOLE1 7, enfatiza em seu trabalho que a sociedade, embora já mais tolerante com a gravidez das adolescentes, casadas ou não, mostra ainda atitude mais negativa do que positiva. Aquelas sentem-se isoladas, pois, não conhecendo a opinião dos amigos, pensam nada mais ter em comum com os mesmos.

MARTIM1 2 caracterizou a gravidez como uma "crise de maturidade" que é especialmente complexa para a jovem que ainda está por descobrir sua identidade.

Em síntese, os aspectos acima citados permitem-nos considerar que o início precoce da menarca e o relacionamento sexual mais oportunizado pelo estilo de vida moderno, cresce cada vez mais o número de adolescentes grávidas e, com elas, os problemas subseq üentes, dos quais alguns serão analisados no presente trabalho.

\section{III - MATERIAL E MÉTODO}

O material da pesquisa foi extraído dos arquivos de um hospitalescola de Porto Alegre, abrangendo o período de maio de 1981 a abril de 1982.

Nesse período deram à luz 1.597 pacientes, das quais 197 situavam-se na faixa etária de 13 até 19 anos, limite por nós considerado para configurar a mãe adolescente.

Foram estudadas as seguintes variáveis: incidência, estado civil, ocupação, época da menarca, in ício das relações sexuais, gestações e paridade, abortamento, métodos anticoncepcionais, freqüência pré-natal e orientações da enfermeira recebidas pela parturiente adolescente. 
MENDES, S.M.A. e Colaboradoras - Gravidez na Adolescência: Atuação da Enfermeira. Rev. Bras. Enf.: RS. 36: 3-12, 1983.

\section{IV - RESULTADOS E DISCUSSŌES}

\section{INCIDENCIA}

Das 1.597 pacientes no período considerado para estudo, 197 eram mães adolescentes, apurando-se uma incidência de $12,39 \%$.

A incidência de mães adolescentes encontrada é bastante elevada quando comparada com a de outros autores que utilizaram limite de idade semelhante ao nosso, tais como: AZNAR \&BENNETT $^{1}$, e KEEVE et alii ${ }^{10}$.

Tabela I - Incidência de adolescentes grávidas segundo a faixa etária

\begin{tabular}{ccr}
\hline IDADE & FREQUENCIA & $\%$ \\
\hline 13 & 2 & 1,01 \\
14 & 3 & 1,52 \\
15 & 10 & 5,07 \\
16 & 19 & 9,64 \\
17 & 36 & 18,27 \\
18 & 60 & 30,45 \\
19 & 67 & 34,01 \\
\hline TOTAL & 197 & 100,00 \\
\hline
\end{tabular}

Tal fato é plenamente justificável, uma vez que no hospital estudado o atendimento abrange considerável número de pacientes previdenciários, o que caracteriza um nível sócio-econômico baixo e, como sabemos, a incidência de mães adolescentes é maior nas classes menos favorecidas, o que pode ser comprovado através do estudo de VITIELLO et alii ${ }^{21}$ e SIQUEIRA ${ }^{20}$.

\section{ESTADO CIVIL}

Tabela II - Estado civil das adolescentes grávidas estudadas, segundo a faixa etária

\begin{tabular}{ccccr}
\hline DADE & \multicolumn{2}{c}{ CASADA } & \multicolumn{2}{c}{ SOLTEIRA } \\
& F & $\%$ & \multicolumn{1}{c}{ F } & $\%$ \\
\hline 13 & 0 & - & 2 & 100,00 \\
14 & 1 & 33,33 & 2 & 66,67 \\
15 & 1 & 10,00 & 9 & 90,00 \\
16 & 5 & 26,31 & 14 & 73,68 \\
17 & 17 & 47,22 & 19 & 52,77 \\
18 & 36 & 60,00 & 24 & 40,00 \\
19 & 36 & 53,73 & 31 & 46,27 \\
\hline TOTAL & 96 & \multicolumn{3}{l}{101} \\
$\%$ & 48,73 & \multicolumn{3}{c}{51,27} \\
\hline
\end{tabular}

O índice de solteiras por nós encontrado parece-nos altamente significativo.

Até os 16 anos, a incidência de mães solteiras é de $79,41 \%$ e $20,59 \%$ de casadas, o que demonstra uma incidência bem maior de solteiras. Talvez isto se deva ao fato de o Código Civil Brasileiro não permitir o casamento da mulher antes dos 16 anos.

Em contrapartida, à medida que aumenta a idade, as casadas e as solteiras se equiparam, havendo uma pequena elevação no número de casamentos, muito embora o de solteiras ainda seja muito elevado, no total dos partos sendo de $51,27 \%$. Este índice deve ser atribuído ao despreparo, ignorância e baixo nível sócio-econômico, características próprias da clientela em estudo. As reações à mãe solteira variam muito de uma cultura para outra, sendo ela aceita em nosso meio com muitas restrições. Entre as famílias que não aceitam a ilegitimidade, o resultado será o casamento forçado, aborto il egal, desgraça social com discriminação legal e social para a criança fruto desse nascimento. Cabe 
MENDES, S.M.A. e Colaboradoras - Gra videz na Adolescência: Atuação da Enfermeira. Rev. Bras. Enf. : RS. 36: 3-12, 1983.

aqui salientar que, segundo BURST 4 e BILLUNG-MEYER ${ }^{3}$, casamentos forçados são instáveis, porque sofrem maiores problemas econômicos e psicológicos, o que explica a taxa elevada de divórcios que os caracteriza; ademais, não sendo a adolescente casada, ficam reduzidas suas chances de casamento futuro.

\section{OCUPAÇÃO}

Tabela III - Ocupação das adolescentes grávidas segundo a faixa etária

\begin{tabular}{cccccc}
\hline IDADE & DO LAR & ESTUDANTE & DOMESTICA & AUX. ESC. & OUTROS \\
\hline 13 & 1 & 1 & - & - & - \\
14 & 3 & - & - & - & - \\
15 & 8 & 2 & - & - & - \\
16 & 15 & 3 & - & - & 1 \\
17 & 26 & 3 & 1 & 3 & 3 \\
18 & 34 & 4 & 6 & 1 & 15 \\
19 & 41 & 2 & 4 & 2 & 18 \\
\hline TOTAL & 128 & 15 & 11 & 6 & 37 \\
$\%$ & 64,97 & 7,61 & 5,60 & 3,04 & 18,78 \\
\hline
\end{tabular}

Das 197 adolescentes estudadas $64,97 \%$ são do lar, o que significa que são economicamente dependentes, quer sejam da família tradicional ou da nova família, o que trará problemas relacionados ao nível sócio-econômico, com a chegada de mais um elemento no grupo familiar, enfatizando mais uma vez que a clientela estudada foi de nível sócio econômico baixo.

Após o parto, o quadro econômico se altera significativamente, pois muito poucas daquelas que têm os seus filhos continuam a viver em suas casas e a serem sustentadas pelos pais. Não é de surpreender, além disso, que estas adolescentes depois do parto tenham menos condições de ganharem seu sustento do que tinham antes (OLSON ${ }^{13}$ ), devido ao encargo dos cuidados com seu bebê.

Para BURST ${ }^{4}$, o problema projeta-se muito além da gravidez em si, influenciando uma sociedade inteira e as culturas que ela contém. Em adição a estas conseqüências, a gravidez precoce restringe oportunidades de a mulher melhorar sua condição sócio-econô mica e bem estar da sociedade.

\section{MENARCA E O INICIO DAS RELAÇÕES SEXUAIS}

Em nosso trabalho, verificou-se que a menarca ocorreu em torno dos 12 - 13 anos, o que traduz um percentual de $51,11 \%$ das adolescentes estudadas, e que coincide com estudos de outros autores, tais como SILVA 19 e VITIELLO ${ }^{21}$.

Das 173 adolescentes em que foi verificado o registro do início das relações sexuais, 107 $(61,85 \%)$ referiram que tiveram sua primeira relação sexual com 16 anos ou menos, fato que demonstra a importância que deve ser dada à educação sexual precoce, para evitar a primeira gravidez na adolescência. BURST ${ }^{4}$ sugere que não seja usado o termo educação sexual, pois este possui conotação limitada, freqüentemente despertando ansiedade e objeção por parte dos pais e líderes religiosos, que acreditam que o conteúdo da educação virá violar tradições, leis ou ensinamentos religiosos, criando promiscuidade. $\mathrm{O}$ termo sugerido pelo autor é promoção de saúde à vida familiar, por ser menos ameaçador e possuir um conceito mais abrangente.

Não devemos salientar somente tópicos sexuais, fisiologia reprodutiva e métodos contraceptivos, mas principalmente a vida familiar, a paternidade, uma vez que a gravidez na adolescência não é só responsabilidade da mulher, o relacionamento interpessoal, o encorajamento do diálogo franco com os pais e um tomar de decisões responsáveis com relação ao comportamento sexual e outras escolhas a serem feitas na vida. 
MENDES, S.M.A. e Colaboradoras - Gravidez na Adolescência: Atuação da Enfermeira. Rev. Bras. Enf.: RS. 36: 3-12, 1983.

\section{GESTAÇŌES E PARIDADE}

Tabela IV - Idade materna correlacionada ao número de gestações e paridade

\begin{tabular}{ccccccccc}
\hline IDADE & I/0 & I/0 & II/1 & III/0 & II/1 & III/2 & IV/2 & SR \\
\hline 13 & 2 & - & - & - & - & - & - & - \\
14 & 3 & - & - & - & - & - & - & - \\
15 & 10 & - & - & - & - & - & - & - \\
16 & 18 & 1 & - & - & - & - & - & - \\
17 & 31 & 4 & - & - & - & - & - & 1 \\
18 & 48 & 6 & 6 & - & - & - & - & - \\
19 & 41 & 13 & 5 & 3 & 1 & 3 & 1 & - \\
\hline TOTAL & 153 & 24 & 11 & 3 & 1 & 3 & 1 & 1 \\
$\%$ & 77,66 & 12,78 & 5,58 & 1,52 & 0,50 & 1,5 & 0,50 & 0,50 \\
\hline
\end{tabular}

No total das 197 adolescentes gestantes, $77,66 \%$ eram primogesta, $18,36 \%$, secundigesta, e multigesta $4.04 \%$, havendo 1 caso $(0,50 \%)$ sem registro de gestação e paridade.

Até os 15 anos, só foram encontrados caso de primigestas e, a partir dos 16 anos, com o aumento da idade, aumentou a paridade. A problemática de gravidez reincidente foi descrita por HOLLINGSWORTH ${ }^{8}$, que no seu estudo comprovou que $26 \%$ de mães adolescentes tinham 2 ou mais filhos, antes dos 20 anos. Salienta-se aqui que, na idade de 19 anos, encontramos inclusive uma mãe que estava na sua 4 a gestação, tendo tido dois partos a termo e um aborto anterior. A gravidade deste caso estende-se não só ao número de gestações em si, mas também à sobrecarga física e emocional ocasionada pela gravidez. Sabe-se que ainda mais graves serão as seqüelas tanto para a mãe quanto para o filho quando todas estas ocorreram durante a adolescência.

\section{ABORTAMENTO}

Das adolescentes estudadas, com mais de uma gestação, registraram-se 17 casos de abortamento $(8,71 \%)$, sendo $4,10 \%$ destes provocados e $4,61 \%$ espontâneos.

PINTO ALEIXO16 encontrou, em um estudo realizado em hospital do Rio de Janeiro, um percentual de abortamentos, provocados ou não, de $2,2 \%$ para as adolescentes e de $6,6 \%$ para o grupo de controle.

Estes resultados nos levam a refletir sobre esta problemática. Talvez, aqui em nosso meio, a pressão social sobre a adolescente grávida seja maior que em outras cidades do país, levando estas jovens mais freqüentemente a optarem pelo aborto.

\section{MÉTODOS ANTICONCEPCIONAIS}

Tabela V - Distribuição das adolescentes segundo a utilização e tipo de métodos anticoncepcionais

\begin{tabular}{cccc}
\hline IDADE & SIM & NÃO & ANTICONCEPCIONAL ORAL * \\
\hline 13 & - & - & - \\
14 & - & 1 & - \\
15 & 1 & 5 & 3 \\
16 & 3 & 6 & 6 \\
17 & 6 & 16 & 12 \\
18 & 12 & 24 & 22 \\
19 & 25 & 75 & 44 \\
** TOTAL & 47 & 61,48 & 93,6 \\
\% & 38,52 & & \\
\hline
\end{tabular}

* 3 pacientes que utilizaram métodos anticoncepcionais não possuiam registro do tipo dos mesmos.

* 75 pacientes não possuiam registro sobre uso de métodos anticoncepcionais. 
MENDES, S.M.A. e Colaboradoras - Gravidez na Adolescência: Atuação da Enfermeira. Rev. Bras. Enf.: RS. 36: 3-12, 1983.

Das 122 adolescentes em que foi pesquisado uso de método anticoncepcional, $75(61,48)$ não faziam uso do mesmo, constituindo uma cifra, embora inferior à encontrada por SILVA ${ }^{19}$, bastante importante quando se refere a adolescentes.

$\mathrm{O}$ que nos chamou mais atenção no resultado do estudo sobre este tópico foi que, das 47 adolescentes que utilizavam anticoncepcional antes da gestação, 44 delas u savam anticoncepcional oral sendo que das três restantes, não foi registrado o método utilizado. Isto pode sugerir que a grande divulgação e a facilidade de obtenção daqueles anticoncepcionais sejam responsáveis pelo seu uso preferencial.

Uma das razões da não utilização de métodos anticoncepcionais, pela maioria das adolescentes, talvez esteja ligada, segundo trabalho realizado por SILVA ${ }^{19}$, ao medo que as adolescentes têm de prejudicarem sua saúde, ou pelo fato de elas se considerarem imunes ao perigo da gravidez. Estes dados também concordam com os de FIELDING ${ }^{7}$-São multiplas as razões do uso inadequado de contraceptivos entre adolescentes: muitas não o utilizavam porque duvidam de sua fecundidade, outras porque não mantêm regularmente vida sexual ativa, e outras por desconhecimento do seu próprio corpo, não ligando o ato sexual à gravidez, segundo HOLLINGSWORTH ${ }^{8}$-Outro fato constatado por SILVA $^{19}$ é que $39 \%$ das entrevistadas em seu trabalho não demonstraram desejo de utilizar anticoncepcional após o parto, o que pode sugerir que a conduta sexual que culminou com a gravidez parece basear-se num forte desejo de desafio, de demonstrar-se pertencente à categoria de adultos, aceitando o perigo e as conseqüências de sua conduta.

Conforme dados da OMS14, estudos recentes, obtidos nos EUA e no Reino Unido, indicam que é relativamente pequena a percentagem de adolescentes sexualmente ativas que usam contraceptivos com regularidade. A ignorância da fisiologia da reprodução e das conseqüências das relações sexuais, por parte de muitas delas, e o fato de que a contracepção segue sendo uma fonte de dificuldades para elas, parecem constituir alguns dos obstáculos principais que se opõem a um uso mais adequado da contracepção na adolescência. Os jovens não discutem facilmente a questão com seus pais e, em muitos casos, estes não se sentem suficientemente preparados para abordar este tema com adolescentes. $\mathrm{O}$ medo de que os pais ou mais velhos saibam que mantêm relações sexuais contribui para a resistência da adolescente em falar sobre comportamento sexual e reprodutor.

\section{FREQÛENCIA PRÉ-NATAL}

Tabela IV - Distribuição da freqüência pré-natal segundo a faixa etária e o trimestre da primeira consulta.

\begin{tabular}{|c|c|c|c|c|c|}
\hline \multirow[b]{2}{*}{ IDADE } & \multirow[b]{2}{*}{ SIM } & \multicolumn{4}{|c|}{ TRIMESTRE DA 1 a CONSULTA * } \\
\hline & & NĀO & 10 & 20 & 30 \\
\hline 13 & 2 & - & & & \\
\hline 14 & 3 & - & - & 1 & - \\
\hline 15 & 9 & 1 & 1 & 1 & 2 \\
\hline 16 & 15 & 3 & 2 & - & 1 \\
\hline 17 & 33 & - & 2 & 2 & 2 \\
\hline 18 & 46 & 4 & 3 & 12 & 3 \\
\hline 19 & 56 & 2 & 7 & 7 & 3 \\
\hline TOTAL * * & 164 & 10 & 15 & 23 & 11 \\
\hline$\%$ & 94,26 & 5,75 & 30,61 & 46,94 & 22,45 \\
\hline
\end{tabular}

Das adolescentes estudadas, 94,26\% fizeram pré-natal, sem no entanto constar nos registros o número de consultas feitas durante a gravidez. A grande maioria delas freqüentou serviços de assistência pré-natal, em postos de atendimento da previdência social, o que evidencia mais uma vez o nível sócio-econômico baixo da população em estudo. Das que não fizeram controle, 6 (60\%) tinham de 18 a 19 anos. Este fato evidencia que, apesar de terem mais idade, não tinham maior conhecimento sobre a importância do acompanhamento pré-natal. Outro fator de não realizarem acompanhamento é devido à freqüente necessidade de ocultarem a gravidez.

* 155 pacientes não possuiam registro sobre o trimestre em que iniciaram a assistência prénatal.

** 23 pacientes não possuiam registro da freqüência pré-natal. 
MENDES, S.M.A. e Colaboradoras - Gravidez na Adolescência: Atuação da Enfermeira. Rev. Bras. Enf.: RS. 36: 3-12, 1983.

Em 115 adolescentes estudadas não foi registrado qual o trimestre da 1 a consulta, o que revela a falta de valorização em termos de qualidade de assistência pré-natal por parte das pessoas que trabalham na área da saúde, que as admitiram e mantiveram contato durante o período de internação, demonstrando também desinteresse pela periodicidade da referida assistência. Das que têm registro 34 $(69,39 \%)$ procuraram o serviço a partir do 29 ou 3 \% trimestre da gravidez, o que impediu em muitas vezes a ação preventiva do profissional. Este fato talvez se justifique, pois, muitas vezes, a preocupação com a gravidez só se evidencia com o crescimento do ventre, o que faz as adolescentes procurarem o atendimento pré-natal tardiamente.

\section{ORIENTAÇŌES DA ENFERMEIRA}

Tabela VII - Distribuição de adolescentes parturientes de acordo com a faixa etária, segundo recebimento de orientações da enfermeira

\begin{tabular}{ccccc}
\hline IDADE & COM REGISTRO & $\%$ & SEM REGISTRO & $\%$ \\
& $\mathrm{~F}$ & & $\mathrm{~F}$ & \\
\hline 13 & 2 & 100,00 & - & - \\
14 & - & - & 3 & 100,00 \\
15 & 5 & 50,00 & 5 & 50,00 \\
16 & 10 & 52,63 & 9 & 47,36 \\
17 & 23 & 63,88 & 13 & 36,11 \\
18 & 34 & 56,66 & 26 & 43,33 \\
19 & 35 & 52,23 & 32 & 47,76 \\
\hline TOTAL & 109 & & 88 & \\
$\%$ & 55,33 & & 44,67 & \\
\hline
\end{tabular}

Encontramos o elevado número de $88(44,67 \%)$ adolescentes que não receberam orientações da enfermeira durante os momentos que antecederam o parto, o que nos chamou muito a atenção, já que daí se pode inferir que as adolescentes em questão não tiveram contato com a enfermeira durante o trabalho de parto. Conhecendo-se as necessidades emocionais de toda paciente sobre o desconhecido, que vem a ser o parto e seu filho, a adolescente com certeza apresenta maiores temores, levando-se em conta a sua imaturidade física e emocional, todos os problemas que pela pouca idade traz consigo e mais os fatores familiares, sociais e econômicos.

Segundo CLARK ${ }^{5}$, o parto é universalmente uma experiência de crise para qualquer muther, podendo vir a ser para a adolescente, devastadora.

Assim sendo, a enfermeira no trabalho de parto deve permanecer junto da adolescente para que esta se sinta estar em contato com uma pessoa interessada, sentindo-se mais segura.

A adolescente jamais deverá ser deixada sozinha, pois isto lhe trará uma sensação de abandono, sentimento que geralmente ela já possui. A enfermeira, não podendo permanecer com a adolescente todo o tempo, devido à atenção a ser dada às outras pacientes, deverá proporcionar-lhe naquele momento a presença do pai da criança, de um familiar ou de uma amiga. Isto nem sempre é aceito nos hospitais, cabendo, portanto, à enf ermeira ser a porta-voz frente à equipe de saúde, para salientar a importância deste acompanhamento da adolescente num período tão crítico de sua existência, que é o dar à luz. Se conseguirmos integrar o cuidado paterno junto às outras lutas da adolescência, este será um problema tão importante e urgente para os homens como já sabemos ser para as mulheres.

Através desta pesquisa, verificamos que as orientações registradas pela enfermeira no atendimento de adolescentes no centro obstétrico $(79,42 \%)$ foram sobre os itens relacionados abaixo:

- exercícios respiratórios e de relaxamento, rotinas do serviço (tricotomia, enema, sinais vitais, banho), conduta no período expulsivo, posicionamento (decúbito lateral) e conduta no trabalho de parto.

Outros itens foram registrados com frequiência individual não significativa.

Somente em dois casos verificou-se registro enfatizando o atendimento a fatores emocionais quanto ao parto, apresentados pelas adolescentes atendidas.

De acordo com REZENDE ${ }^{18}$, as adolescentes, especialmente sobre o ponto de vista psicológico, precisam de atendimento, pois elas sofrem nitidamente conflitos devido ao medo da dor, vergonha e reprovações dos familiares, ressentindo-se dos impactos mentais.

A sequiência dos diferentes aspectos da assistência de enfermagem durante o trabalho de parto, quer seja por necessidades físicas, emocionais ou de observação, segundo ZÍEGEL ${ }^{22}$, não se en- 
MENDES, S.M.A. e Colaboradoras - Gravidez na Adolescência: Atuação da Enfermeira. Rev. Bras. Enf.: RS. 36: 3 - $12,1983$.

contra em ordem de prioridade. Para esta autora, orcicisir seria impossível, pois cada trabalho de parto difere dos outros, assim como a resposta üi cada adolescente. Assim sendo, as prioridades de atendimento serão determinadas pelas condições maternas e fetais.

A enfermeira pode of erecer apoio emocional através da confiança que transmite, de suas atitudes e maneiras de agir, mesmo quando apressada, frente a um parto iminente. É necessário ter bom senso, em cada um dos casos, para decidir qual a melhor seqüência na assistência de enfermagem à adolescente.

O sucesso nos relacionamentos de apoio da enfermeira com a adolescente, conforme CLARK $^{5}$, é influenciado por sua própria estrutura psicobiológica. A obtenção de um relacionamento satisfatório com a mãe adolescente depende, sem dúvida, de sua habilidade em avaliar seu próprio comportamento em relação a ela.

É sabido que a mulher em trabalho de parto requer uma presença quase constante de uma pessoa simpática, compreensível e acessível junto dela, alguém que lhe transmita um interesse pessoal, tanto pelas ações quanto por palavras.

Embora em nosso estudo tenhamos verificado que as orientações da enfermeira foram basicamente técnicas, as mesmas são importantes não só para fornecer conforto físico como também demonstram interesse da enfermeira pela adolescente, sendo este, talvez, uma das mais efetivas formas de que disponha para ajudar.

\section{V - CONCLUSŌES E RECOMENDAÇŌES}

A incidência de $12,39 \%$ de adolescentes grávidas no hospital em estudo merece, por parte da enf ermeira, uma atenção especial.

$\mathrm{O}$ Índice de solteiras de $51,30 \%$ por nós encontrado, parece altamente significativo. Para reduzir de maneira substancial a freqüência de gravidez extra-matrimonial necessitaremos esforços conjugados por parte da equipe de saúde. $O$ grande número de adolescentes grávidas somente com profíssão do lar $(64,97 \%)$, em nosso estudo, mostra a dependência econômica desta populaçầo, chamando a atenção para o problema social existente.

Os resultados por nós encontrados referentes à menarca incidiram na faixa etária de 12 a

13 anos.

Em $61,21 \%$ dos casos, as adolescentes estudadas ref eriram que sua primeira relação sexual foi aos 16 anos ou menos, o que demonstra a precocidade da jovem adolescente na iniciação sexual.

As gestações e paridade mostram que um total de $77,66 \%$ das adolescentes eram primigesta, $18,36 \%$ secundigesta e $4,04 \%$ multigesta.

$\mathrm{O}$ número de abortamentos verificados no grupo em estudo foi de $8,71 \%$, sendo que $4,10 \%$ foram provocados e 4,61\% espontâneos. Embora em nosso meio o aborto seja ilegal, as pressões sociais as levam a interromper a gravidez indese jada.

Das adolescentes estudadas, $61,48 \%$ não faziam uso de métodos anticoncepcionais. A anticoncepção não é assunto liberado entre pais e filhos e, mesmo nas escolas, ainda é tabu.

A maioria das adolescentes $(94,25 \%)$ freqüentou serviços de assistência pré-natal, mas somente $30,61 \%$ procuraram o serviço no primeiro trimestre de gravidez, $46,94 \%$ procuraram no segundo trimestre e $22,45 \%$ no terceiro trimestre. Por estes dados, podemos concluir que $69,39 \%$ das adolescentes só procuraram os serviços a partir do segundo ou terceiro trimestre, demonstrando por este fato que a ação preventiva nesta população estudada fica prejudicada pelo início tardio do controle pré-natal.

Quanto à atuação da enfermeira em termos assistenciais, encontramos elevado número (44,67\%) de parturientes adolescentes que não receberain orientações nos momentos que antecederam o parto. Das restantes, $79,42 \%$, foram registros que enfocavam mais atividades técnicas, consideradas como importantes para a adolescente grávida principalmente pelos aspectos relacionados ao manuseio de seu corpo e as implicações deste em relação ao parto e seu fillho.

A atitude da enfermeira que atendeu a adolescente pode desempenhar papel importante, caso consiga entender as limitações físicas e psíquicas da jovem, avaliando-a e ajudando-a a enfrentar suas ansiedades e dificuldades no papel de mãe, podendo desta maneira minorar os conflitos emocionais existentes. Consideramos importante que os profissionais da área da saúde avaliem as necessidades da mãe adolescente e, tão logo seja feita esta avaliação, decidam como atendê-la.

Deveriam existir programas e locais específicos para o atendimento de adolescentes grávidas, onde fossem atendidos os pais, o pai da criança e os amigos, que serviriam de apoio às jovens que se encontram em tal situação. 
MENDES, S.M.A. e Colaboradoras - Gravidez na Adolescência: Atuação da Enfermeira. Rev. Bras. Enf.: RS. 36: $3-12,1983$.

Quando os profissionais de saúde usarem todas as habilidades que desenvolvem, isto é, avaliação, aconselhamento e ensino em combinação com encaminhamentos apropriados, é possível que muita mães adolescentes possam ser auxiliadas a encontrar uma vida mais rica e mais satisfatória.

\section{SUMMARY}

The present work was elaborated emphasizing our concern with the world-wide problem of the teenager with regard mainly to the increasing frequency of teenager pregnancy in other countries, as well as with the lack of specific assistance to this group in Porto Alegre. To this effect a retropesctive study was made, based on 1.597 case histories of expectant women assisted in a teaching Hospital in our city, among whom the convincing evidence of 12.39 per cent pregnant adolescents was found. It has been our intention, through this work, to characterize the problem posed by the pregnant teenager in relation to: Matital status, occupation, age for menarche, beginning of sexual intercourse, pregnancies and parity, abortion, contraceptive methods, prenatal visits and nursie performance in providing care for the pregnant adolescent.

\section{VI - REFERENCIAS BIBLIOGRĀFICAS}

1 - AZNAR, Ramon \& BENNET, A. E. Pregnancy in the adolescent girl. Am. J. Obst. e Ginec., Ohio, 81 (5): 934-40, 1981 .

2 - BABIKIAN, Hrair \& GOLDMAN, A. Study in teenage pregnancy. Amer. J. Psychiat., Washington, DC, 128 (6): 111-6, 1971.

3 - BILLUNG - MEYER, Jo. The single mother: Can we help? The Canadian Nurse, Winnipeg, $26: 8$, nov. 1979.

4 - BURST, Helen V. Adolescent pregnancies and problems. Joumal of Nurse-Midwifery, Charleston, Southcaroline, 24:2, march/april 1979.

5 - CLARK, Ann L. The crisis of adolescent unwed motherhood. American Journal of Nursing, St. Francisco, California, 67:1465-69, July 1967.

6 - CURTIS, Frances L. S. Observation of unwed pregnant adolescents. American Journal of Nursing, New York, 74 (1): 100-2, 1974.

7 - FIELDING, Jonathan. Adolescent pregnancy revisited. Massachusetts Departament of Public Health, Washington St., Boston, 299 (16): 893-6, oct. 1978.

8 - HOLLINGSWORTH, Doroty R. The pregnancy adolescent. In: Adolescent Obstetric and Gynecology. Chicago, Year Book Medical Publishers, 1980. Chapter 4, p. 67-7.

9 - HUFFMAN, Dewhurst Capraro. Anatomy and physiology. In: The gynecology of childhood and adolescent. 2 a ed. Philadelphia, W. B. Saunder Company, 1981. Chapter 1, p. 24-69.

10-KEEVE, J. Philip \& SCHLESINGER, Edward R. Fertility experience of juvenil girls: a communit wide ten-year study. Am. J. Ph. 59:2185-97, 1969.

11-KLAUS, Hanna. Experience with teenage pregnancy. Bull. Amer. Coll. Nu rse Midwives, Washington, 17(4): 114-121, 1972.

12-MARTIM, C. Psychological problems of abortion for the unwed teenage. Genet Psychol Mong. 88 (1): 23-110, 1973 .

13-OLSON, Lucy. Social and psychological correlates of pregnancy resolution among adolescent women: a rewiew. American Orthopsychiatric Association. Washington, DC. 50(3): jul. 1980.

14-ORGANIZAÇĀO PANAMERICANA DE LA SALUD. Série de Informes Técnicos. Embarazo y el aborto na adolescência. Washington, 1975. p. 7-28. (Publicacion Cientifica, 538).

15-PIERRE, Tena S. \& PIERRE, Richard. Adolescent pregnancy: guidelines for a comprehinsive shool-based program. Health Education, Pennsylvania, 11(3): 12-3, may/june 1980.

16-PINTO ALEIXO, Pedro. Gestação na adolescência. Jornal Brasileiro de Ginecologia, Rio de Janeiro, $91(6): 439-42$, nov./dez. 1981.

17-POOLE, Carole J. Assessing the needs of the adolescent mother. Washington state journal of nursing. Washington, Seattle, 51(2): 27-34, Summer/Fall 1979.

18-REZENDE, Jorge \& PERRICELLI, Francisco. Primiparidade precoce e tardia. Rev. de Ginecologia e Obstetrícia, Brasil, 46(5): 305-24, maio 1953.

19-SILVA, João L. Pinto e et alii. Gravidez na adolescência - I conduta frente a anticoncepção e ao sexo. Jornal Brasileiro de Ginecologia, Rio de Janeiro, 90(6): 283-7, dez. 1980. 
MENDES, S.M.A. e Colaboradoras - Gravidez na Adolescência: Atuação da Enfermeira. Rev. Bras. Enf.: RS. 36: $3-12,1983$.

20

ço pré-natal do Centro de Saúde Geraldo de Paula Souza, São Paulo (Brasil). Revista de Saúde Pública, São Paulo, 15(5): 449-54, out. 1981.

21 -VITTIELLO, Nelson et alii. Assistência pré e perinatal à mãe adolescente. Jornal Bras. de Ginecologia, São Paulo, 85(5): 229-35, 1977.

22-ZIEGEL, Erna. Assistência Clínica durante o Trabalho de Parto. In: Enfermagem Obstétrica. 7. ed. Rio de Janeiro, Interamericana, 1980 . cap. 14, p. 235-74. 